\title{
ANÁLISIS MULTIDISCIPLINAR DE LA OSTEOGÉNESIS IMPERFECTA
} MULTIDISCIPLINARY ANALYSIS OF IMPERFECT OSTEOGENESIS

Broch Michavila, A.; Gimbel Benítez, S.; Seguiel Correa, F. y *Arráez Aybar, Luis A. Facultad de Medicina. Medicina y Terapia Ocupacional. Universidad Complutense de Madrid. *Facultad de Medicina. Universidad Complutense de Madrid. alexbrochmi@gmail.com

\section{RESUMEN}

La Osteogénesis imperfecta, es una enfermedad hereditaria que causa un déficit en la formación de colágeno tipo I. El manejo de la osteogénesis imperfecta debe ser multidisciplinar, se utilizan tratamientos y terapias farmacológicas, físicas, quirúrgicas y psicológicas.

Palabras Clave: osteogenesis imperfecta; osteogenesis imperfecta \& occupational therapy; osteogenesis imperfecta heart; osteogenesis imperfecta ribs; osteogenesis imperfecta diagnosis.

\section{SUMARY}

Imperfect osteogenesis is a hereditary disease that causes a deficit in the formation of type I collagen. The management of osteogenesis imperfecta must be multidisciplinary, pharmacological, physical, surgical and psychological treatments and therapies are used.

Key words: Imperfect osteogenesis; Imperfect osteogenesis \& occupational therapy; Imperfect osteogenesis heart; Imperfect osteogenesis ribs; Imperfect osteogenesis diagnosis. 


\section{INTRODUCCIÓN}

La Osteogénesis Imperfecta (OI), actualmente entra dentro de la clasificación de las llamadas “enfermedades raras” por su baja incidencia, ya que afecta a 1 de cada 10.000 recién nacidos (puede variar según fuentes), asimismo, afecta a mujeres y a hombres sin distinción de sexos ni razas en el mundo.

Afecta principalmente a la correcta formación de colágeno (tipo I habitualmente), lo que produce una fragilidad congénita del hueso; consecuentemente se producen distintos signos y síntomas que pueden ir desde un escaso número de fracturas, hasta deformidades severas que pueden aumentar el riesgo de muerte.

Habitualmente se aborda con medidas conservadoras, mediante farmacoterapia (con bifosfonatos) e incluso con correcciones quirúrgicas de las deformidades en casos avanzados y/o peligrosos.

La OI se puede definir como una enfermedad hereditaria que tiene la característica común de presentar fragilidad congénita del hueso. Puede ser autosómica dominante, recesiva o de novo. Las formas leves de OI, pueden llegar a manifestarse con deformidades óseas, dolores complicados de manejar, provocando una limitación funcional importante para las Actividades de la Vida Diaria (AVDs), así como entorpeciendo y dificultando un desarrollo normal en ámbitos personales y sociales.

\section{MANIFESTACIONES CLÍNICAS}

La OI es una enfermedad cuya principal manifestación clínica son las lesiones óseas como las fracturas y osteoporosis. Por ello, el médico debe realizar un seguimiento exhaustivo de los marcadores de actividad ósea tanto en suero como en orina de forma regular. Aunque ésta sea la principal presentación clínica, los profesionales de la salud deben estar al corriente de que las patologías secundarias a estas lesiones son de gran importancia. Esto es debido a que la enfermedad no es letal en sí misma, pero su pronóstico se ve afectado por todas las lesiones colaterales, por lo que su tratamiento es principalmente multidisciplinar.

En cuanto a la patología ósea se ha demostrado que existe una asociación clara (26\% de los pacientes con OI) entre la OI y sufrir escoliosis y anormalidades craneales y vertebrales. Estas 
patologías óseas incluyen impresión basilar, invaginación basilar y platibasia. Dado su riesgo potencial para producir malformaciones (por ejemplo: Arnold-Chiari), es de vital importancia su seguimiento y control. En contra, se ha demostrado que algunas enfermedades del tejido conectivo como la hiperlaxitud articular, pese a ser muy frecuente (70\% de los pacientes con OI), son un pobre predictor de la evolución de la enfermedad, y que son independientes de la severidad de la OI.

Las lesiones secundarias (no óseas) más frecuentes en la OI que ponen en riesgo la vida del paciente son las alteraciones en el sistema cardiopulmonar. El manejo médico se centra en minimizar la morbilidad de las fracturas, dolor y deformidades de los huesos mediante la maximización de la salud ósea con la optimización del nivel de vitamina D y la ingesta de calcio junto con la terapia ocupacional.

\section{CALIDAD DE VIDA}

Como hemos hablado anteriormente, las intervenciones clínicas e investigaciones se han centrado principalmente en los resultados ortopédicos, genéticos y farmacológicos de las personas con OI y aunque la calidad de vida ha ganado reconocimiento como un factor importante de los pacientes, ha recibido poca atención en los individuos con esta enfermedad. La calidad de vida física en niños y adultos con OI parece ser menor que la de la población general. Por otro lado, la calidad de vida mental y psicosocial es igual o mejor en los individuos con OI que la de la población general.

Dolor, escoliosis, limitaciones de la actividad son restricciones debido a la disminución de la función física, se asocian con niveles más bajos de la calidad de vida funcional. Los investigadores deben ponerse de acuerdo sobre una definición de la calidad de vida en lo que respecta a las medidas de OI y uso validado apropiado para la evaluación de la calidad de vida en OI.

Se evaluó un programa de transición para los adolescentes y jóvenes adultos con OI de un hospital de ortopedia. Este estudio confirmó que un modelo de talla única de transición para los pacientes con OI sería inapropiado dentro de las instituciones. Deben aprovecharse las oportunidades para crear programas a la medida de transición, en teoría, con las preferencias del paciente, sobre todo las de los adultos jóvenes. 
En este mismo sentido, Oppenheim (6), observó que las deformidades de la columna vertebral, resultan difíciles de corregir debido a su fragilidad, al no poder ser toleradas las fuerzas correctoras ejercidas por los corsés. El uso de corsés se limitaría únicamente a evitar futuras deformidades más que la corrección en sí; aún con riesgo de aumentar un compromiso respiratorio previamente existente.

No obstante, las órtesis más que actuar como un sustituto de la musculatura débil (a causa de la inmovilidad, por riesgo de fractura), ofrece mayor seguridad a los pacientes y a sus familias, facilitando la participación, la inclusión y con ellos, la independencia funcional, mejorando progresivamente la fuerza muscular. Este proceso, a su vez, previene la osteoporosis al aumentar la densidad ósea; es por esta razón que los huesos de los miembros superiores "usados y estresados" en las AVDs, son más densos que aquellos menos empleados; presentando menor riesgo de fractura (7).

En este caso, los autores de la investigación (5), especifican que la órtesis confeccionada con material termoplástico tipo polipropileno o polietileno con distintos grosores en función de la zona a contener, sirven para controlar y minimizar la rotación de la articulación en cuestión ayudando a descargar el hueso, consiguiendo parcialmente alivio del dolor. Su uso, será continuo durante el día a excepción de la noche y en períodos de tratamiento.

\section{CONCLUSIÓN}

Jette, afirma que "si la investigación clínica en terapias físicas ampliase su búsqueda de resultados de investigación clínica a una explícita e hipotética relación entre el deterioro y la discapacidad, los resultados estimularían un cambio en el paradigma de investigación dominante en la profesión... ....dicho cambio en el paradigma de la investigación en terapias físicas, podría producir resultados que tengan un impacto directo en la práctica clínica”. En pocas palabras, la investigación es mínima, para el amplísimo campo donde se debe trabajar. Las terapias farmacológicas y quirúrgicas son un hecho y tienen buenos resultados; no obstante, la terapia física se debe beneficiar del tratamiento ortésico como terapia coadyuvante para la mejora de la capacidad funcional del individuo. Un paciente no es sólo un paciente, es una persona que cumple un rol social que se ve afectado por un proceso patológico como la OI, abarcar todos los aspectos necesarios, desde la interdisciplinariedad 
estricta y confinada dará como resultado, sin lugar a dudas, una mejora en la calidad de vida del paciente y de su familia.

\section{BIBLIOGRAFÍA}

Hoyer-Kuhn H., Semler O., Stark C., Struebing N., Goebel O., Schoenau E. A specialized rehabilitation approach improves mobility in children with osteogenesis imperfecta. $\mathrm{J}$ Musculoskelet Neuronal Interact 14(4), 445-453, 2014.

L Hill C., O Baird W., J Walters S. Quality of life in children and adolescents with Osteogenesis Imperfecta: a qualitative interview based study. Research.

H.H. Engelbert R., A. Beemer F., van der Graaf Y., J.M. Helders P. Osteogenesis Imperfecta in Childhood: Impairment and Disability - A follow-up Study. Archives of Physical Medicine and Rehabilitation, 80, 896-903, 1999.

Bishop N. Characterising and treating osteogenesis imperfecta. Early Human Development, 86, 743-746, 2010.

I.M. Alguacil Diego, F. Molina Rueda, M. Gómez Conches. Tratamiento ortésico en pacientes con osteogénesis imperfecta. An Pediatr (Barc). 2011;74(2):131.e1-131.e6

Oppenheim W. The spine in osteogenesis imperfecta: a review of treatment. Connect Tissue Res. 1995:31:S59-63.

Cole WG. Upper extremity problems in OI: Think function or cosmesis. Proceedings of the $7^{\text {th }}$ International Conference on Osteogenesis Imperfecta. Canada: Montreal; 1999. 